BNL-112104-2016-JA

\author{
TLD efficiency calculations \\ for heavy ions: an analytical approach
}

Daria Boscolo, Emanuele Scifoni, Antonio Carlino, Chiara La Tessa, Thomas Berger, Marco Durante, Valeria Rosso, and Michael Kramer

Submitted to the European Physical Journal D

December 2015

Collider Accelerator Department

Brookhaven National Laboratory

\author{
U.S. Department of Energy \\ USDOE Office of Science (SC), \\ Nuclear Physics (NP) (SC-26)
}

Notice: This manuscript has been authored by employees of Brookhaven Science Associates, LLC under Contract No. DE- SC0012704 with the U.S. Department of Energy. The publisher by accepting the manuscript for publication acknowledges that the United States Government retains a non-exclusive, paid-up, irrevocable, world-wide license to publish or reproduce the published form of this manuscript, or allow others to do so, for United States Government purposes. 


\section{DISCLAIMER}

This report was prepared as an account of work sponsored by an agency of the United States Government. Neither the United States Government nor any agency thereof, nor any of their employees, nor any of their contractors, subcontractors, or their employees, makes any warranty, express or implied, or assumes any legal liability or responsibility for the accuracy, completeness, or any third party's use or the results of such use of any information, apparatus, product, or process disclosed, or represents that its use would not infringe privately owned rights. Reference herein to any specific commercial product, process, or service by trade name, trademark, manufacturer, or otherwise, does not necessarily constitute or imply its endorsement, recommendation, or favoring by the United States Government or any agency thereof or its contractors or subcontractors. The views and opinions of authors expressed herein do not necessarily state or reflect those of the United States Government or any agency thereof. 


\title{
TLD efficiency calculations for heavy ions: an analytical approach
}

\author{
Daria Boscolo ${ }^{1,2}$, Emanuele Scifoni ${ }^{1 a}$, Antonio Carlino ${ }^{1,3,4}$, Chiara La Tessa ${ }^{5}$, Thomas Berger ${ }^{6}$, Marco Durante ${ }^{1}$, \\ Valeria Rosso $^{2}$, and Michael Krämer ${ }^{1}$ \\ 1 GSI Helmholtzzentrum für Schwerionenforschung GmbH, Darmstadt, Germany. \\ 2 Department of Physics, University of Pisa, Pisa, Italy. \\ 3 EBG MedAustron GmbH, Wiener Neustadt, Austria. \\ 4 Department of Physics and Chemistry, University of Palermo, Palermo, Italy \\ 5 Brookhaven National Laboratory (BNL), Upton NY, United States. \\ ${ }^{6}$ German Aerospace Center (DLR), Institute of Aerospace Medicine,Cologne, Germany.
}

Received: date / Revised version: date

\begin{abstract}
The use of thermoluminescent dosimeters (TLDs) in heavy charged particles' dosimetry is limited by their non-linear dose response curve and by their response dependence on the radiation quality. Thus, in order to use TLDs with particle beams, a model that can reproduce the behavior of these detectors under different conditions is needed. Here a new, simple and completely analytical algorithm for the calculation of the relative TL-efficiency depending on the ion charge $Z$ and energy $E$ is presented. The detector response is evaluated starting from the single ion case, where the computed effectiveness values have been compared with experimental data as well as with predictions from a different method. The main advantage of this approach is that, being fully analytical, it is computationally fast and can be efficiently integrated into treatment planning verification tools. The calculated efficiency values have been then implemented in the treatment planning code TRiP98 and dose calculations on a macroscopic target irradiated with an extended carbon ion field have been performed and verified against experimental data.
\end{abstract}

PACS. XX.XX.XX No PACS code given

\section{Introduction}

Thermoluminescence dosimeters (TLDs) are passive solid state detectors widely used in conventional (photon) radiation detection and dose verification [1]. The development of ion beam cancer therapy, and the research in radioprotection in space, stimulated the use of the TLDs also for heavy ion dosimetry [2]. The main advantages of this kind of detector, compared, e.g., to ionization chambers, are the small dimensions, relative ease of handling, little interference with the radiation field and the usability in solid state phantoms. However, the non-linear dose response curve of TLDs represents a limitation for heavy-ion dosimetry. It results, indeed, in a dependence of the detector response on the radiation quality and on the particles LET (Linear Energy Transfer) [3,4]. Unlike photon radiation, which creates a nearly uniform dose distribution on a microscopic level, heavy ions generate an extremely localized dose deposition along the trajectory of the primary ion reaching extremely high values. Thus, assuming that a TLD is characterized by a linear-supralinear-saturated (or even sub-linear) dose-response curve, when irradiated with heavy charged particles the detector will saturate in the region surrounding the track core, then it will respond in

\footnotetext{
a e-mail: e.scifoni@gsi.de
}

a supra-linear way for the intermediate distances and linearly for the external part of the track (Fig. 1). Therefore, in order to use TLDs for ion beams dosimetry, and specifically to get a prediction of their response in a treatment plan irradiation, it is necessary to develop a model able to compute the relative effectiveness of these detectors for different ions and a broad range of different energies.

Several models describing the TLDs response to ion beams already exist $[1,4-9]$ and most of them are based on a heavy computational effort. This work describes an alternative analytical solution and, in particular, it focuses on a method based on the Local Effect Model (LEM) [10]. Even though LEM was originally developed for predicting the response of biological systems following ion irradiation, it can also be extended for efficiency calculations of solid state detectors, such as TLDs [1,11-13]. This model uses an amorphous track structure and requires the knowledge of both the radial dose distribution around heavy ion trajectory and the detector response to a reference radiation. In this context, an algorithm was developed [1] for computing efficiency tables for heavy charged particle radiation fields. Although this approach has been well received thanks to its simplicity and the good agreement with experimental data, some limits hindering its robustness have been suggested [14]. 

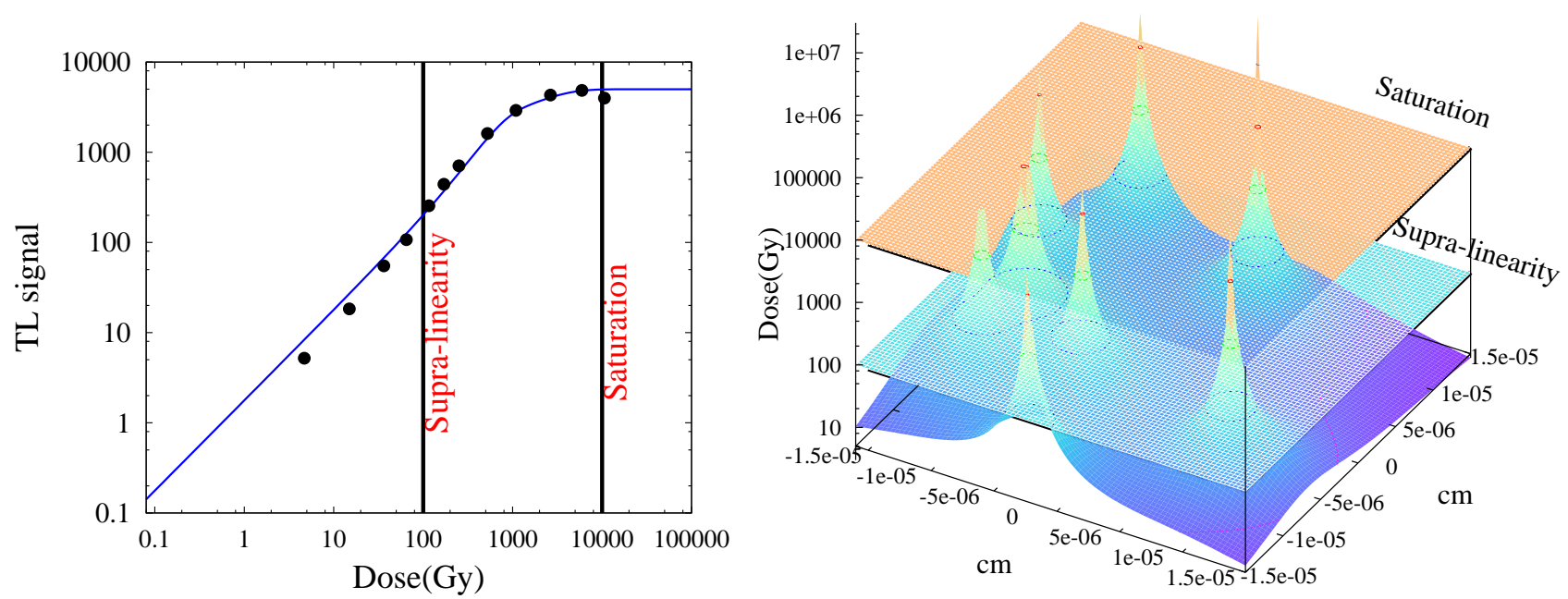

Fig. 1. Effect of the non-linearity in the dose-response curve for a TLD-700 ( $\left.{ }^{7} \mathrm{LiF}: \mathrm{Mg}, \mathrm{Ti}\right)$ when irradiated with ion beams. On the left panel: the characteristic dose-response curve for a TLD-700 irradiated with X-rays, exhibiting three different regimes: linear, supra-linear and saturated. Experimental data from [15]. On the right panel: local dose distribution of a $3 \mathrm{MeV}$ proton beam with a fluence of $10^{10} \mathrm{~cm}^{-2}$ impinging on a LiF slab. The local dose distribution crosses all three aforementioned response regimes.

For this reason, a new, simple and completely analytical algorithm for the calculation of the TLD efficiency dependence on ion charge $Z$ and energy $E$ has been developed. The computed efficiency tables have been compared with experimental data for different ion types and have been integrated in treatment planning verification tools in order to calculate the detector response for a macroscopic target irradiation. Experimental measurements have been also performed in order to validate the model.

\section{Relative effectiveness}

The behavior of a dosimeter in a radiation field $H I$ different from the reference radiation field, $X$ can be described through the relative effectiveness $\eta$. In literature the relative effectiveness is not uniquely defined [12], in this work we will refer to the iso-dose definition, i.e., the ratio between the response of the detector irradiated in a radiation field $H I, S_{H I}(D)$, and the response after irradiation with reference radiation $X, S_{X}(D)$, at the same nominal dose, D.

$$
\eta_{i s o-d o s e}=\left.\frac{S_{H I}(D)}{S_{X}(D)}\right|_{i s o-d o s e}
$$

\section{Single Ion Approach}

The Single Ion Approach (SIA) is a basically analytical method to calculate the relative effectiveness of TL detectors according to Eq. 1. This model assumes that each ion contributes to the TLD response independently and hence the detector signal after ion irradiation is calculated by linearly summing up the signal of all the individual ions. This assumption represents the main limitation of the model. Being a LEM-like model, this approach requires as input the knowledge of the radial dose distribution $D(r)$ around a particle path and a parametrization of the lowLET dose-response curve for the dosimeter, $T L(D)$. In this work, the sub-models chosen are the same proposed in [1]. The adopted radial dose distribution as a function of the distance from the ion path $r$ is, indeed, given by the formulation

$$
D(r)=\left\{\begin{array}{lll}
k & \text { if } & r \leq a_{0} \\
k\left(a_{0} / r\right)^{2} & \text { if } & a_{0}<r<r_{\max } \\
0 & \text { if } & r_{\max }<r
\end{array}\right.
$$

where $k$ is a normalization factor, $a_{0}$ is the size of the track core and $r_{\max }$ is the maximum range of secondary electrons. The value of $a_{0}$ is kept fixed and it is of the same order of magnitude as the crystal lattice spacing $\left(a_{0}=1 \AA\right)$.

The main idea, as shown in Fig. 2, is to fold the radial dose distribution of a single particle with the detector lowLET dose-response curve, obtaining the corresponding TL radial distribution $T L(D(r))$.

The signal generated by a single ion, $S_{H I}$, can thus be calculated by integrating the TL radial distribution over its track. For the $T L_{X}(D)$ and of $D_{i}(r)$ functional forms considered in this work, this integral can be solved analytically, avoiding the sampling of the radial dose distribution. This represents a great advantage with respect to previous approaches, such as the grid summation method proposed in [1]; indeed, the sampling of the radial dose distribution is highly inefficient with Monte Carlo methods and represents one of their main limitations [14]. Moreover, as the integration on the dose distribution is not performed directly, but rather on the radial response distribution $T L_{X}(D)$, it results in a smoother and broader 

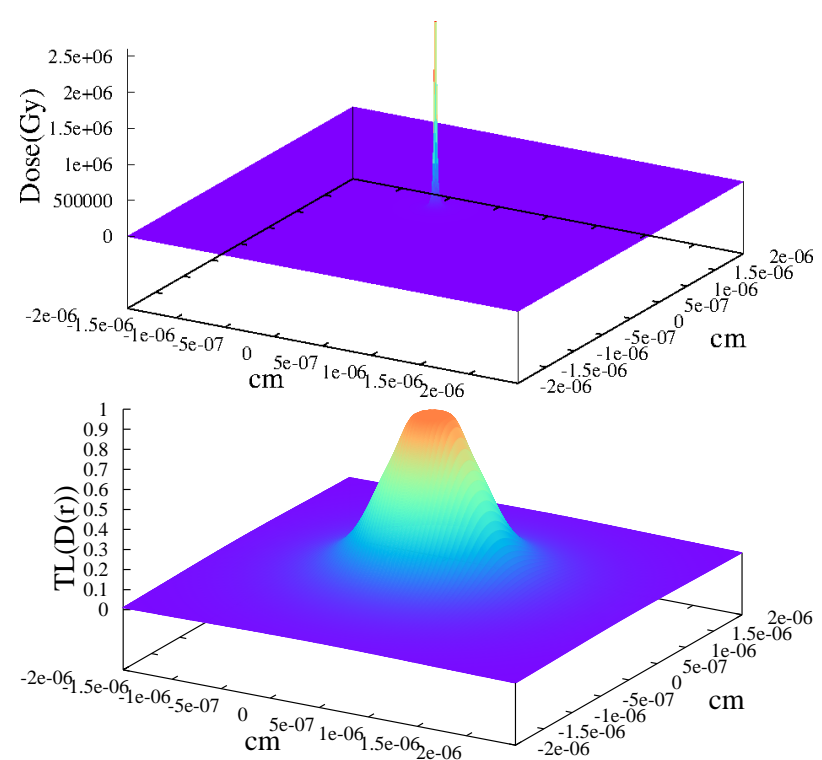

Fig. 2. Radial dose distribution, $D(r)$, (top) and TL response distribution, $T L(D(r))$, (bottom) around a single ion track.

shape on which the sampling is much more effective (see Fig. 2). This is extremely important when using numerical rather than analytical approaches to compute $S_{H I_{i}}$, as it may become necessary for future developments and refinements of the model.

\subsection{Relative effectiveness calculation}

In order to calculate the relative effectiveness for a particle beam of given energy $E$, atomic number $Z$ and fluence $\phi$ a simple algorithm has been developed. The relative effectiveness is calculated for different ion species, as a function of the ion energy, according to Eq. 1. X-ray radiation has been chosen as the low-LET reference radiation. The two quantities $S_{H I}(D)$ and $S_{X}(D)$ are computed separately as described below.

Computation of $S_{H I}$. In order to consider the primary ion slowing down process inside a TLD, the detector is divided into slices normal to the incident particle direction. The slice thickness $\Delta t_{i}$ is chosen so that the primary ion energy variation inside the layer is constant within $1 \%$. The energy loss in each detector slice is computed with the use of stopping power tables obtained from the radiation transport code ATIMA [16].

For each slice $\left(\Delta t_{i}\right)$ the TL radial distribution $T L_{X}\left(D_{i}(r)\right)$ around a single ion is calculated. As mentioned above, the signal produced by the ion on the $i$-th slice can be computed as

$$
S_{H I_{i}}=2 \pi \Delta t_{i} \int_{0}^{r_{\max _{i}}} T L_{X}\left(D_{i}(r)\right) r d r,
$$

where $r_{\text {max }_{i}}$ is the maximum range of secondary electrons emitted by the primary ion. In this work $r_{\max _{i}}$ is computed as in ECLaT [1]. To obtain the signal $S_{H I}$ produced by a single ion, the contribution of all the slices is summed up:

$$
S_{H I}=\sum_{i=0}^{\# \text { slices }} S_{H I_{i}}
$$

Computation of $S_{X}$. In this case the dose is assumed to be uniformly distributed over the whole detector. The total dose deposited by the ion beam of energy $E$, charge $Z$ and fluence $\phi$ is thus calculated as:

$D_{\text {tot }}[\mathrm{Gy}]=1.6 \times 10^{-10} \times \frac{1}{\rho}\left(\frac{\Delta \mathrm{E}}{\Delta \mathrm{T}}\right)\left[\frac{\mathrm{MeV} \mathrm{cm}}{\mathrm{g}}\right] \times \phi\left[\mathrm{cm}^{-2}\right]$.

Here $\Delta T$ is the thickness of the detector, if the particles are so energetic that they can cross the dosimeter; otherwise, if the particles are stopped inside the detector, $\Delta T$ represents their range. $\Delta E$ is the total energy deposited in the TLD.

The signal $S_{X}$ is computed on a small area defined as the area in which, on average, one ion is found. This area is computed as a function of the beam fluence $\phi$ as:

$$
A_{\text {Sim }}=1 / \phi \text {. }
$$

Thus, in analogy with the method adopted in [1], the signal $S_{X}$ is given by

$$
S_{X}\left(D_{t o t}\right)=T L_{X}\left(D_{t o t}\right) \times A_{s i m} \times \Delta L,
$$

where $\Delta L$ is the detector thickness.

\section{Calculation of the detector response for a macroscopic target irradiation with TRiP98}

TRiP98 (TReatment planning for particle) is a code dedicated to the planning of radiotherapy treatments with energetic ions $[17,18]$. Apart from performing dose calculation and optimization, it is also able to predict detector responses in the mixed radiation field generated in a macroscopic target irradiation (for plan verification issues). In particular, a module for simulating the response calculation of solid state dosimeters is implemented [19, 20].

The dosimeter response as a function of the detector position in a mixed field, $\boldsymbol{x}$, can be calculated by combining the nuclear fragmentation spectra, $\frac{d N}{d E}(\boldsymbol{x}, Z, E)$, with the relative effectiveness $\eta\left(Z_{i}, E\right)$, of different ions species, $Z$, and different incident particles energies $E$ as:

$S(\boldsymbol{x})=$ const $\times \sum_{i=1}^{Z_{\text {proj }}} \int_{0}^{E_{0}} \frac{d N}{d E}\left(\boldsymbol{x}, Z_{i}, E\right)\left(\frac{d E}{d x} \frac{1}{\rho}\right)_{i} \eta\left(Z_{i}, E\right) d E$. 

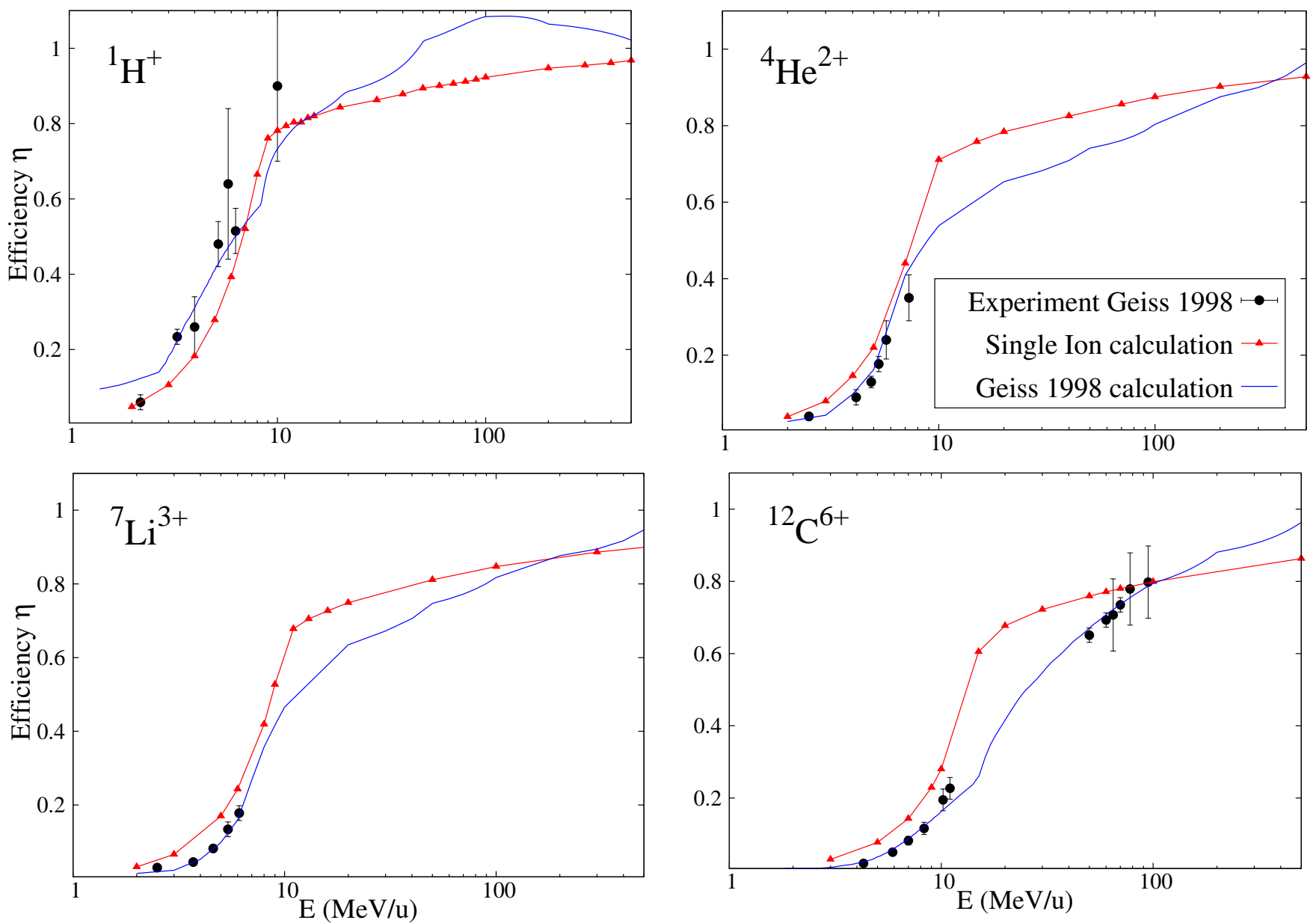

Fig. 3. Single ion efficiency calculations for TLD-700 (red triangles with eye guiding line) compared with ECLaT calculations (blue solid line) and measurements for monoenergetic irradiation [1](full circles) for various ions. The fluence is $10^{7} \mathrm{~cm}^{-2}$ and the detector thickness is $380 \mu \mathrm{m}$.

\section{Experimental verification setup}

To validate the model, dose profiles have been measured with TLDs for different depths in a water phantom irradiated by a fully ionized carbon ion beam. The measurements were performed at GSI (Darmstadt, Germany).

The phantom was filled with water and equipped with mixed materials for studies of different purpose [21]. Here, only the measurements performed in pure water are presented, Fig. 6.

The phantom was irradiated with a treatment plan designed for a $5 \times 3 \times 3 \mathrm{~cm}^{3}$ water target placed at $11 \mathrm{~cm}$ depth. The treatment plan and a virtual CT of the target were generated with TRiP98. The water equivalent thickness of the water phantom has been accounted into the TPS (treatment planning system) considering the different density of the detectors. The smallest beam focus was chosen for each energy (between 3 and $4 \mathrm{~mm}$ FWHM at the isocenter in air) and a distance of $2 \mathrm{~mm}$ between raster points in the orthogonal beam plane and $3 \mathrm{~mm}$ in depth were selected as a fair compromise between an homogeneous dose distribution in the target and a feasible irradiation time. In each configuration the physical dose of a single beam was optimized and computed. The target has been scanned with 29 energies, from a proximal energy of $235.85 \mathrm{MeV} / \mathrm{u}$ to a distal one of $E_{\max }=270.55$ $\mathrm{MeV} / \mathrm{u}$. The prescribed physical dose to the target was 1 Gy.

The dose profile was measured with TLD-700 $\left({ }^{7} \mathrm{LiF}\right.$ : $\mathrm{Mg}, \mathrm{Ti}$ ), of $3.2 \times 3.2 \times 0.9 \mathrm{~mm}^{3}$ size and an average density of $2.635 \mathrm{~g} \mathrm{~cm}^{-3}$. The detectors were provided by the German Aerospace Center (DLR) (Cologne, Germany). The measurements were performed at 16 different water depths and, for each measurement position, the signal was calculated as the mean over 6 dosimeters. The dose calibration in ${ }^{137} \mathrm{Cs}$ radiation and the read-out procedure were performed at DLR and details on the procedure are described in [2]. While the errors associated to the data presented in [1] are mainly due to the uncertainty on CR39 fluence measurement; a relative error of the $10 \%$ has been associated to the data presented in this work considering several independent factors such as: the calibration, the nominal read out uncertainty, the uncertainty on the dose delivered and the averaging over six TLDs. 


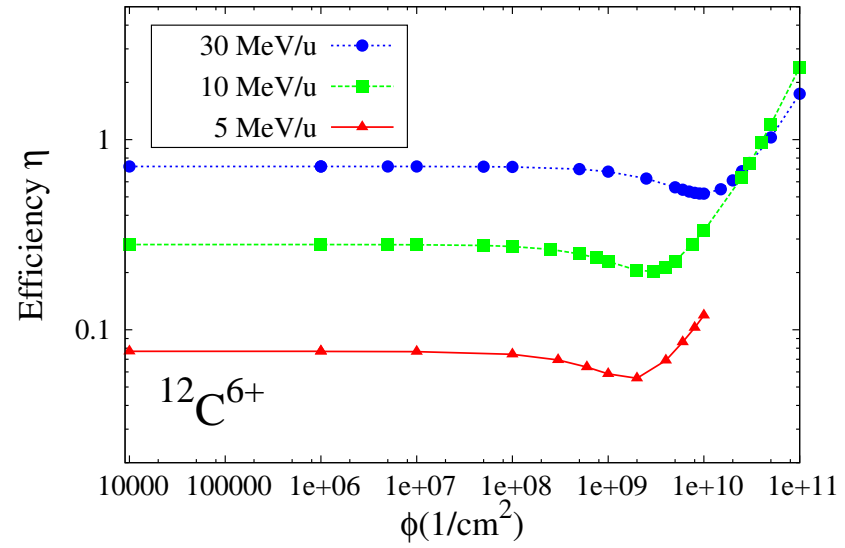

Fig. 4. Relative effectiveness calculation with the single ion approach varying the fluence for carbon ions of different energies.

\section{Results and discussion}

Relative effectiveness tables have been calculated with SIA for different ions and have been compared with the calculated values provided by different approaches, such as the one proposed in [1], as well as with experimental data [1]. These data are consistent with other measurements (taking into account the error bars) [2]. The effectiveness values for fully ionized protons, helium, lithium and carbon ions as a function of the energy per nucleon are reported in Fig. 3. The value appears lower for higher $\mathrm{Z}$ ions and, for the same ion type, the effectiveness decreases for less energetic particles. An increasing linear energy transfer of the particles results in an increase of the ionization density and, thus, the saturation effects are more pronounced $[1,20]$.

However, even though the computed efficiency follows the expected behavior, the agreement with the experimental data is still not optimal and for carbon ions the efficiency values reported in [1] seem to better reproduce the measurements. Furthermore, it is important to remark that the SIA relies on the assumption that each ion contributes independently to the detector response. All the contributions coming from the neighboring tracks are assumed to sum up linearly, and this represents the main assumption (and thus limitation) of the model. For carbon ions, having a more inhomogeneous radial dose distribution, the issue of a non-linear response when tracks are overlapping is more important than for protons. The validity limits have been, nevertheless, analyzed for different fluences and energies.

In Fig. 4 the relative effectiveness has been reported as a function of the fluence for carbon ions of different energies. As expected, for relatively low fluences below $10^{8}$ $\mathrm{cm}^{-2}$, the effectiveness does not change and the single ion approximation is justified. This is also the typical fluence range used in standard clinical treatments with carbon ions. At higher fluence values, the ion tracks get closer and the relative effectiveness curve decreases due to the effect of supra-linearity in the inter-track region. In this

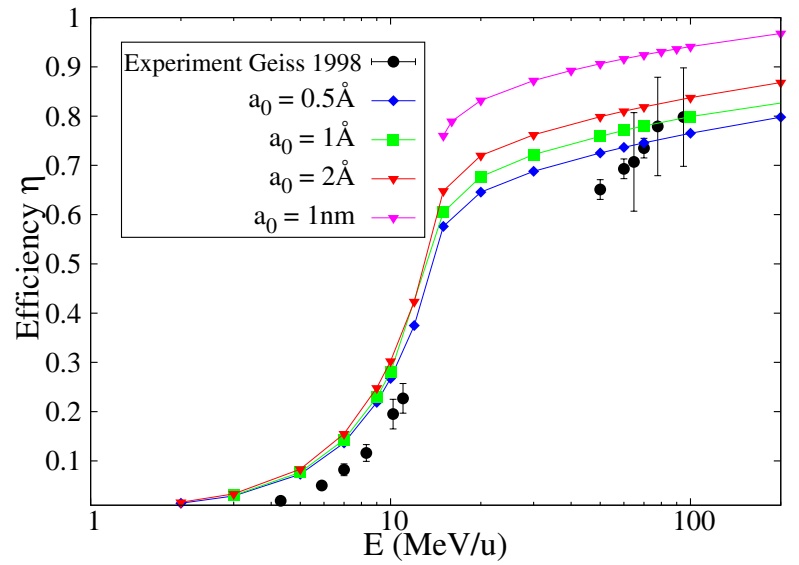

Fig. 5. Impact of the core size, $a_{0}$ on the effectiveness calculation for TLD-700 irradiated with carbon ions.

case, ions can not be considered independent anymore. The increase in the effectiveness for very high fluences is instead due to the saturation of the TL response for the low-LET radiation.

A robustness analysis against changes on the core size, $a_{0}$, of the radial dose distribution has been also performed. Fig. 5 shows the impact of variations of the core size in the calculation of the relative effectiveness for carbon ions. For larger core size the relative effectiveness is larger. Indeed, in that case the core dose decreases and, thus, the saturation effect is less pronounced.

The computed efficiency tables obtained with SIA have been imported into TRiP98 for predicting detector responses in a macroscopic phantom irradiation. In order to compare the calculated responses with the experimental data, we considered an extended water target with the same geometry described in Sect. 5. Fig. 6 shows a comparison between TRiP98 planned dose and the calculated TLD-700 signal response in the beam-eye-view direction

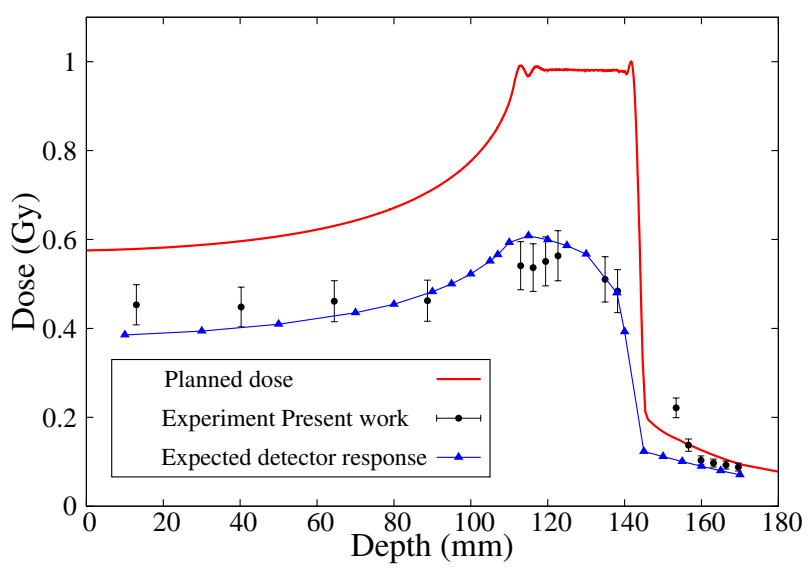

Fig. 6. Depth dose distribution comparison of the planned dose by TRiP98 (red solid line), the computed TLD response calculated importing the efficiency tables provided by the Single Ion approach on TRiP98 (blue triangles with eye guiding line) and the experimental data (black dots) for carbon ion irradiation in a water target. 
computed with the efficiency tables provided by SIA. The experimental data presented in this work are reported too. The plot indicates a good agreement between calculation and measurements in the target volume. However the predicted responses are not able to reproduce the experimental data with enough accuracy in the tail region behind the target volume. In detail, the calculated dose seems to be underestimated compared to the experimental data points especially in the proximity of the target edge. So far, this behavior is unexplained but similar observations have been already reported [22].

\section{Conclusion}

In order to extend the use of TLDs in charged particle fields, accurate efficiency tables must be produced over a broad range of energies and particles. The Single Ion Approach, SIA, represents a new algorithm for calculating relative effectiveness tables for particles with different energy $E$ and charge $Z$. This method computes the detector response starting from its response to a single ion track. A similar approach has been proposed for $\mathrm{Al}_{2} \mathrm{O}_{3}$ : C optically stimulated luminescence detectors [23]. The calculated efficiencies have been compared with experimental data [1] as well as with calculated values provided by different approaches.

The relative effectiveness tables calculated with our model have been implemented in the treatment planning code TRiP98 [18] and signal calculations in macroscopic targets irradiated with an extended carbon ion field were performed. The results have been compared with experimental data performed at GSI (Darmstadt, Germany).

In conclusion, although SIA is at a first stage of development it shows an accuracy level comparable with previous calculations. Moreover, being analytical, it is computationally fast, it can be efficiently integrated in treatment planning verification tools and the agreement with experimental data on macroscopic target irradiation is good. These features represent the basis for further extension, including incorporation of corrections for higher fluence values.

We gratefully acknowledge the help of Dr. S. Greilich and Dr. N. Bassler with numerous discussions. Part of the research leading to these results has received funding from the European Union Seventh Framework Programme [PEOPLE - 2013 - ITN - ARGENT project] under grant agreement n [608163].

D.B., E.S. and M.K. developed the model. A.C. and C.L.T. performed the experiments. T.B. prepared the dosimeters and performed their read out. M.D., V.R. and M.K. coordinated and supervised the work. D.B. wrote the manuscript, and all the authors revised it.

\section{References}

1. O.B. Geiß, M. Krämer, G. Kraft, Nucl. Instrum. Meth. B. 142, 592 (1998)
2. T. Berger, M. Hajek, Radiat. Meas. 43, 146 (2008)

3. Y. Horowitz, Thermochimica Acta 135, 127 (1988)

4. L. Larsson, R. Katz, Nucl. Instrum. Meth. 138, 631 (1976)

5. M. Waligorski, R. Katz, Nucl. Instrum. Meth. 175, 48 (1980)

6. J. Kalef-Ezra, Y. Horowitz, Int. J. Appl. Radiat. Isot. 33, 1085 (1982)

7. P. Olko, Radiat. Meas. 38(46), 781 (2004)

8. P. Olko, Radiat. Meas. 41, S57 (2007)

9. S. Greilich, U. Hahn, M. Kiderlen, C. Andersen, N. Bassler, Eur. Phys. J. D 68(10), 327 (2014)

10. M. Scholz, G. Kraft, Adv. Space Res. 18, 5 (1996)

11. B. Spielberger, M. Scholz, M. Krämer, G. Kraft, Phys. Med. Biol. 46(11), 2889 (2001)

12. R. Herrmann, S. Greilich, L. Grzanka, N. Bassler, Radiat. Meas. 46(12), 1551 (2011)

13. E. Scifoni, Mod. Phys. Lett. A, in press (2015)

14. N. Bassler, Ph.D. thesis, Department of Physics and Astronomy, Faculty of Science, University of Aarhus (2006)

15. B. Majborn, L. Bøtter-Jensen, P. Christensen, On the Relative Efficiency of TL Phosphors for High-Let Radiation.Proceedings of the 5th International Conference on Luminescence Dosimetry (1977), pp. 124-130

16. GSI, unpublished. http://web-docs.gsi.de/ weick/atima/ 17. M. Krämer, M. Scholz, Phys. Med. Biol. 45, 3319 (2000)

18. M. Krämer, O. Jäkel, T. Haberer, G. Kreft, U. Weber, Phys. Med. Biol. 45, 3299 (2000)

19. B. Spielberger, M. Scholz, M. Krämer, G. Kraft, Phys. Med. Biol. 47(22), 4107 (2002)

20. B. Spielberger, M. Krämer, G. Kraft, Phys. Med. Biol. 48(4), 497 (2003)

21. A. Carlino, Master's thesis, Scuola di Specializzazione in Fisica Medica, Università degli studi di Palermo (2012)

22. O. Geiß, Ph.D. thesis, University GhK Kassel, Germany (1997)

23. G. Sawakuchi, E.G. Yukihara, Phys. Med. Biol. 57, 437 (2012) 